\section{(1) \\ CrossMark}

\title{
Time for a longer and better life for patients with COPD
}

\author{
Lowie E.G.W. Vanfleteren ${ }^{1,2}$, Anders Ullman ${ }^{3}$ and Leonardo M. Fabbri $\mathbb{1}^{3,4}$
}

Affiliations: ${ }^{1}$ Research and Development, CIRO, a Centre of Expertise for Chronic Organ Failure, Horn, The Netherlands. ${ }^{2}$ Respiratory Medicine, Maastricht University Medical Hospital, Maastricht, The Netherlands. ${ }^{3}$ COPD Centre, Sahlgrenska University Hospital, Gothenburg, Sweden. ${ }^{4}$ Dept of Medicine, University of Ferrara, Italy.

Correspondence: Leonardo M. Fabbri, COPD Centre, Sahlgrenska University Hospital, University of Gothenburg, Vita Stråket 12, 41345 Göteborg, Sweden. E-mail: leonardo.michele.fabbridgu.se

@ERSpublications

Managed care may reduce mortality in severe COPD patients with previous hospitalisation http://ow.ly/THpI30hiaYJ

Cite this article as: Vanfleteren LEGW, Ullman A, Fabbri LM. Time for a longer and better life for patients with COPD. Eur Respir J 2018; 51: 1702569 [https://doi.org/10.1183/13993003.02569-2017].

Together with the adequate delivery of the right drugs to the right patient at the right dosage, and aimed at the right outcomes, the most important management goals in patients with chronic obstructive pulmonary disease (COPD) are the following: smoking abstinence, physical activity and a balanced diet; adhering to complex medication regimens; and developing the ability to recognise signs and symptoms of deterioration, and knowing what to do to prevent or reverse them [1].

In recent years a number of multicomponent (self) management programmes have been studied in patients with COPD, but results have been variable and even equivocal, most likely because of variations in the intensity, duration, delivery and content of the programmes [2,3]. Furthermore, the programmes have been directed to optimise only the management of COPD and not that of concomitant chronic diseases. COPD is one of the most frequent and important components of chronic multimorbidity caused by, or associated with, smoking, inactivity and ageing, i.e. with the most important risk factors of multimorbidity [4]. Hence, COPD is seldom seen alone [5]. The improvement in quality of life achieved with the management of COPD as a single disease is limited to the reduction of symptoms and exacerbations but, at variance with other individual chronic diseases, has resulted in no effect on the progression of COPD or the survival of patients [1].

Globally, hospitalisations [6], particularly re-hospitalisations [7], for multimorbidities present major challenges for the patient and for the healthcare provider. Although major advancements have been made in the management of acute events (e.g. myocardial infarction [8]) and in the chronic management of some individual components of multimorbidity (e.g. heart failure [9]), with significant improvement in quality of life and reduction of complications, hospitalisations and mortality, little if any progress has been made in the management of patients with chronic multimorbidity [10]. While managed care, particularly self-management, has been proven to be useful in most individual chronic diseases (e.g. better in heart failure and diabetes, less so in asthma and COPD) [11], no proper study has been conducted in patients with multimorbidities.

Published online Jan 11 2018; republished Jan 172018 with an amendment to figure 1.

Received: Dec 112017 | Accepted: Dec 122017

Conflict of interest: Disclosures can be found alongside this article at erj.ersjournals.com

Copyright CERS 2018 
In this issue of the European Respiratory Journal, two new studies on the effectiveness of managed care programmes in patients with COPD have been published: the COMET study [12] and the PIC-COPD study [13]. Both studies describe a multicomponent self-management intervention to reduce healthcare utilisation during a 1-year follow-up. The primary outcome in the COMET study was the number of unplanned all-cause hospitalisation days; in the PIC-COPD study, the number of emergency department visits. The COMET study specifically adapted the programme for patients with very severe and complex COPD. The PIC-COPD study targeted patients with moderate-to-severe COPD and at least two significant concomitant chronic diseases.

Although both studies reported negative results for the primary outcome, they showed a striking effect on mortality, the most important secondary outcome. We believe that, when two independent, well-designed, well-conducted studies have negative results for the primary outcome but report strongly positive results for an outcome as important as mortality, it is imperative to use these results to design and conduct properly powered studies with the primary outcome of mortality, particularly for diseases such as COPD that are not modified by current pharmacological treatment.

Although previous studies with self-management interventions in COPD have not been able to show an effect on survival [2,3] and, in one study there was even an increased mortality [14], we may still speculate about what could have made the difference in the studies presented here.

Most likely, the answer lies in the inclusion of a high-risk population, i.e. with severe COPD, at least one hospitalisation in the previous year, and significant multiple concomitant chronic diseases. Indeed, the COMET study consisted of a very complex COPD population with multiple comorbidities and a high risk of death: 76\% were GOLD D patients, 43\% had at least MMRC grade 3, 44\% had BODE index scores $>7$, $75 \%$ were on long-term oxygen therapy, $25 \%$ were on noninvasive ventilation, and, on average, patients had 3.5 concomitant diseases. The impact of these comorbid diseases is clear, because less than half of the 313 hospitalisations were due to COPD exacerbations. In the PIC-COPD study, an older population with milder COPD than in the COMET study, patients had at least two comorbidities, $75 \%$ had cardiovascular disease, and the intervention was aimed at improving the self-management not only of COPD but also of concomitant diseases. One large study demonstrated that a simple disease management programme may reduce hospitalisations and emergency department visits but not mortality [15]; this study was conducted in a very similar population with severe COPD and previous hospitalisations, and the patients who, at variance with all other studies, were almost exclusively men recruited through Veterans Affairs hospitals. However, 2 years later, a similar study conducted by the same group had to be interrupted for (unexplained) increased mortality in the self-management arm [14].

Past trials often excluded COPD patients with significant comorbid disease or did not prioritise management of treatable comorbid disease. Patients with multimorbid COPD are particularly vulnerable to care fragmentation because of the need to access care from various healthcare disciplines and professions, and the potential for receiving conflicting medical advice [16]. Both studies delivered health services with integrated care, communication with, for example, e-health platforms or case managers, and transmission of information to hospital physicians or family physicians, and access to ambulatory outpatient clinics.

The lack of effect on hospitalisation could be explained by the studies being underpowered. In the COMET study, statistically significant differences between groups were observed for all-cause acute care hospital days in the per-protocol analysis. In the PIC-COPD study, difference in emergency department visit risk was seen, for those at risk, i.e. those with previous emergency department visits. However, the contrast between the lack of effect on hospitalisation and the improved survival might be explained by a better awareness and recognition of deterioration of symptoms achieved by a self-management intervention, which might indeed result in earlier hospitalisation, more appropriate treatment and, thus, better outcome. Indeed, although a similar number of hospitalisations were seen in COMET, most deaths in that study were related to COPD exacerbations.

Exacerbations can be complex, and outcome may be related to exacerbations of concomitant diseases [17]. Prognostic models in COPD did not include exacerbations in their model. They included airflow obstruction, dyspnoea, functional capacity and age [18, 19] but ,importantly, also extrapulmonary manifestations such as low BMI [18] and comorbidities [20-22]. Thus, taking into account the multimorbid population in both studies, self-management programmes that also aim to take into account the management of comorbidities might be successful in reducing mortality. This remains to be shown in a study properly designed and powered for mortality as a primary endpoint. An important limitation of both studies is that they did not properly assess adherence to treatment, a potentially important risk factor for reduced disease control, hospitalisation and, eventually, survival. Furthermore, it is not mentioned and/or documented how the self-management programs also took into account the management of comorbidities. 


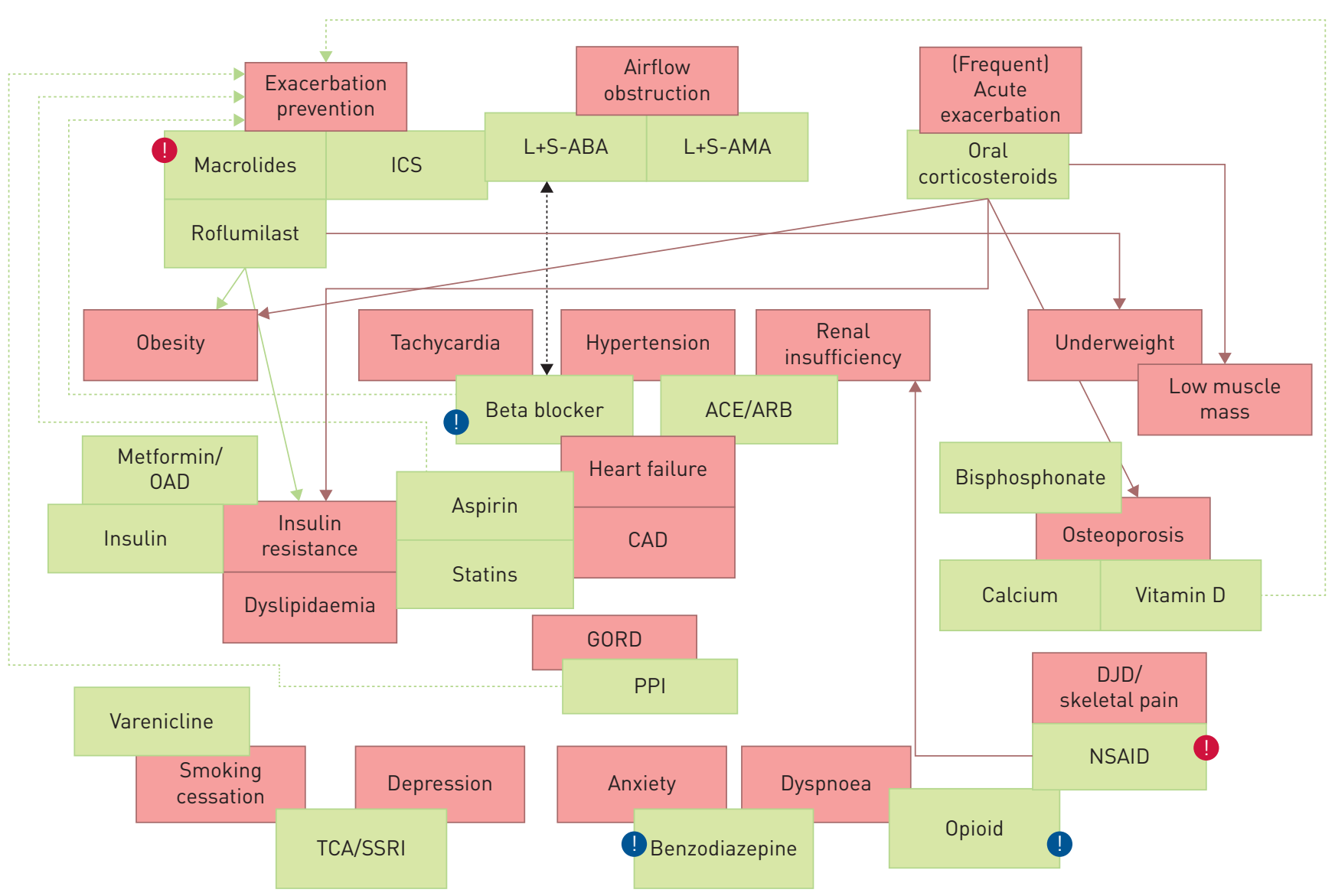

FIGURE 1 The pharmacological perspective for an integrated management of chronic obstructive pulmonary disease (COPD) and its comorbidities. Patients with multimorbidity are more likely to have multidrug therapies. Treatments for these multiple conditions may interfere with each other, and complex medication regimens in multimorbid patients increase the risk of adverse events and difficulties with compliance. One such complexity may arise when a drug that improves one chronic disease is found to worsen another. In these scenarios, clinicians often have to set priorities in managing patients with multimorbidity, which is challenging. On the other hand, cross-beneficial effects have also been described, such as a potential beneficial effect of proton pump inhibitors or beta-blockers for prevention of exacerbation, or improved insulin sensitivity through the use of roflumilast. This emphasises the potential of integration of the management of COPD and its comorbidities. This figure illustrates the most prevalent conditions and most common drugs in patients with COPD and the potential positive and negative interactions; it is not intended to be comprehensive. ICS: inhaled corticosteroids; L+S-ABA: long- and short-acting beta-agonists; L+S-AMA: long- and short-acting muscarinic antagonists; ACE/ARB: angiotensin-converting enzyme inhibitor/angiotensin-receptor blocker; OAD: oral antidiabetic drug; CAD: coronary artery disease; GORD: gastro-oesophageal reflux disease; PPI: proton pump inhibitor; DJD: degenerative joint disease; TCA: tricyclic antidepressants; SSRI: selective serotonin reuptake inhibitors; NSAID: nonsteroidal anti-inflammatory drugs. Red box: medical condition; green box: drug; black arrow: interaction; green arrow: potential positive influence; red arrow: potential negative influence; full arrow: well-documented effect; dotted arrow: potential effect; red exclamation mark: potential associated cardiovascular risk; blue exclamation mark: potential associated respiratory risk.

Because both studies had negative results for primary outcome, secondary outcomes can be considered only for generating hypotheses. However, in COPD, for which no pharmacological treatment improves survival [1], the consistent effect on mortality of these two studies is striking and may provide a strong rationale for designing and powering a new study for COPD and concomitant chronic diseases, with mortality selected as primary outcome. Indeed, we know that scientific evidence is obtained only by designing new studies after a detailed evaluation of secondary outcomes and post hoc analyses of previous negative studies. Two clear examples of this are roflumilast in COPD [23, 24] and mepolizumab in severe asthma $[25,26]$. In both cases, the original studies conducted in general populations were negative [23, $25]$, but later studies in selected subgroups of patients were clearly positive [24, 26].

Despite important progress in the pharmacological treatment of patients with COPD, which aims at improving lung function, reducing symptoms, improving quality of life, and preventing exacerbations, a large proportion of patients still progress to severe COPD with multiple comorbidities and frequent, unpredictable exacerbations of COPD or concomitant diseases [17]. Although COPD exacerbations can be reduced, no drug has yet been shown to improve survival by reducing exacerbations [1]. 
For a long time, apart from smoking cessation, long-term oxygen therapy for severe hypoxia, and volume reduction surgery in specific emphysematous patients, no other intervention has been shown to prevent progression or improve survival of COPD patients [1].

Lately, things are changing for the better, particularly in patients with severe COPD. Home nocturnal noninvasive ventilation in patients with persistent hypercapnic respiratory failure has been shown to improve survival and reduce re-hospitalisations in COPD patients who remain hypercapnic after an acute exacerbation [27]. Triple inhaled treatment with a combination of long-acting anticholinergics, beta-adrenergics and steroids has been shown to further improve quality of life and reduce exacerbations in patients whose disease is not controlled by other options [28]; furthermore, there is reasonable hope that triple therapy might reduce mortality. Treatment of concomitant chronic diseases is associated with decreased hospitalisations and increased survival, but this evidence is based only on registry data [29]. However, the rationale for the integrated management of comorbidities of COPD, even if complex, is particularly strong (figure 1) [30].

In this issue of the journal are two important management studies consistently showing a considerable reduction of mortality in patients comprehensively treated with management plans that address not only the COPD component but also the complexity of multimorbidities [12,13]. We believe it is time to conduct a properly designed and powered study with the goal of combining all these positive observations to achieve a better quality of life for patients with severe multimorbidities. Let's move this forward.

\section{References}

1 Vogelmeier CF, Criner GJ, Martinez FJ, et al. Global Strategy for the Diagnosis, Management, and Prevention of Chronic Obstructive Lung Disease 2017 Report: GOLD Executive Summary. Eur Respir J 2017; 49 : 1700214.

2 Lenferink A, Brusse-Keizer M, van der Valk PD, et al. Self-management interventions including action plans for exacerbations versus usual care in patients with chronic obstructive pulmonary disease. Cochrane Database Syst Rev 2017; 8: CD011682.

3 Jonkman NH, Westland H, Trappenburg JC, et al. Characteristics of effective self-management interventions in patients with COPD: individual patient data meta-analysis. Eur Respir J 2016; 48: 55-68.

4 Barnes PJ. Senescence in COPD and its comorbidities. Annu Rev Physiol 2017; 79: 517-539.

5 Vanfleteren LE, Spruit MA, Groenen M, et al. Clusters of comorbidities based on validated objective measurements and systemic inflammation in patients with chronic obstructive pulmonary disease. Am $J$ Respir Crit Care Med 2013; 187: 728-735.

6 Muka T, Imo D, Jaspers L, et al. The global impact of non-communicable diseases on healthcare spending and national income: a systematic review. Eur J Epidemiol 2015; 30: 251-277.

7 Lindenauer PK, Dharmarajan K, Qin L, et al. Risk trajectories of readmission and death in the first year following hospitalization for COPD. Am J Respir Crit Care Med 2017; in press [https://doi.org/10.1164/rccm. 201709-1852OC].

8 Anderson JL, Morrow DA. Acute myocardial infarction. N Engl J Med 2017; 376: 2053-2064.

9 Metra M, Teerlink JR. Heart failure. Lancet 2017; 390: 1981-1995.

10 Fabbri E, Zoli M, Gonzalez-Freire M, et al. Aging and multimorbidity: new tasks, priorities, and frontiers for integrated gerontological and clinical research. J Am Med Dir Assoc 2015; 16: 640-647.

11 Hanlon P, Daines L, Campbell C, et al. Telehealth interventions to support Self-management of long-term conditions: a systematic metareview of diabetes, heart failure, asthma, chronic obstructive pulmonary disease, and cancer. J Med Internet Res 2017; 19: e172.

12 Kessler R, Casan-Clara P, Koehler D, et al. COMET: a multicomponent home-based disease-management programme versus routine care in severe COPD. Eur Respir J 2018; 51: 1701612.

13 Rose L, Istanboulian L, Carriere L, et al. Program of Integrated Care for Patients with Chronic Obstructive Pulmonary Disease and Multiple Comorbidities (PIC COPD ${ }^{+}$): a randomised controlled trial. Eur Respir J 2018; 51: 1701567.

14 Fan VS, Gaziano JM, Lew R, et al. A comprehensive care management program to prevent chronic obstructive pulmonary disease hospitalizations: a randomized, controlled trial. Ann Intern Med 2012; 156: 673-683.

15 Rice KL, Dewan N, Bloomfield HE, et al. Disease management program for chronic obstructive pulmonary disease: a randomized controlled trial. Am J Respir Crit Care Med 2010; 182: 890-896.

16 Vanfleteren LE, Spruit MA, Franssen FM. Tailoring the approach to multimorbidity in adults with respiratory disease: the NICE guideline. Eur Respir J 2017; 49: 1601696.

17 Beghe B, Verduri A, Roca M, et al. Exacerbation of respiratory symptoms in COPD patients may not be exacerbations of COPD. Eur Respir J 2013; 41: 993-995.

18 Celli BR, Cote CG, Marin JM, et al. The body-mass index, airflow obstruction, dyspnea, and exercise capacity index in chronic obstructive pulmonary disease. N Engl J Med 2004; 350: 1005-1012.

19 Puhan MA, Garcia-Aymerich J, Frey M, et al. Expansion of the prognostic assessment of patients with chronic obstructive pulmonary disease: the updated BODE index and the ADO index. Lancet 2009; 374: 704-711.

20 Divo M, Cote C, de Torres JP, et al. Comorbidities and risk of mortality in patients with chronic obstructive pulmonary disease. Am J Respir Crit Care Med 2012; 186: 155-161.

21 Divo MJ, Martinez CH, Mannino DM. Ageing and the epidemiology of multimorbidity. Eur Respir J 2014; 44: 1055-1068.

22 Divo MJ, Casanova C, Marin JM, et al. COPD comorbidities network. Eur Respir J 2015; 46: 640-650.

23 Calverley PM, Sanchez-Toril F, McIvor A, et al. Effect of 1-year treatment with roflumilast in severe chronic obstructive pulmonary disease. Am J Respir Crit Care Med 2007; 176: 154-161.

24 Calverley PM, Rabe KF, Goehring UM, et al. Roflumilast in symptomatic chronic obstructive pulmonary disease: two randomised clinical trials. Lancet 2009; 374: 685-694. 
25 Flood-Page P, Swenson C, Faiferman I, et al. A study to evaluate safety and efficacy of mepolizumab in patients with moderate persistent asthma. Am J Respir Crit Care Med 2007; 176: 1062-1071.

26 Pavord ID, Korn S, Howarth P, et al. Mepolizumab for severe eosinophilic asthma (DREAM): a multicentre, double-blind, placebo-controlled trial. Lancet 2012; 380: 651-659.

27 Murphy PB, Rehal S, Arbane G, et al. Effect of home noninvasive ventilation with oxygen therapy vs oxygen therapy alone on hospital readmission or death after an acute COPD exacerbation: a randomized clinical trial. JAMA 2017; 317: 2177-2186.

28 Singh D, Vestbo J. Triple therapy in chronic obstructive pulmonary disease. Am J Respir Crit Care Med 2017; 196: 1082-1083.

29 Mancini GB, Etminan M, Zhang B, et al. Reduction of morbidity and mortality by statins, angiotensin-converting enzyme inhibitors, and angiotensin receptor blockers in patients with chronic obstructive pulmonary disease. $J \mathrm{Am}$ Coll Cardiol 2006; 47: 2554-2560.

30 Vanfleteren L, Spruit MA, Wouters EFM, et al. Management of chronic obstructive pulmonary disease beyond the lungs. Lancet Respir Med 2016; 4: 911-924. 